\title{
Comparaison de l'utilisation des ressources forestières et de la régénération entre deux types de forêts claires à Isoberlinia au Togo
}

MARRA DOURMA ${ }^{\mathbf{1}}$

KPÉRKOUMA WALA 1

Ronald Bellefontaine 2

Komlan BataWILA ${ }^{1}$

Kudzo Atsu Guelly ${ }^{1}$

Koffi AKpaganA ${ }^{1}$

1 Laboratoire de botanique et écologie végétale Faculté des Sciences Université de Lomé BP 1515, Lomé

Togo

2 Cirad

Département Systèmes biologiques

Unité propre de recherche Diversité génétique TA A-39/C

34398 Montpellier Cedex 5 France

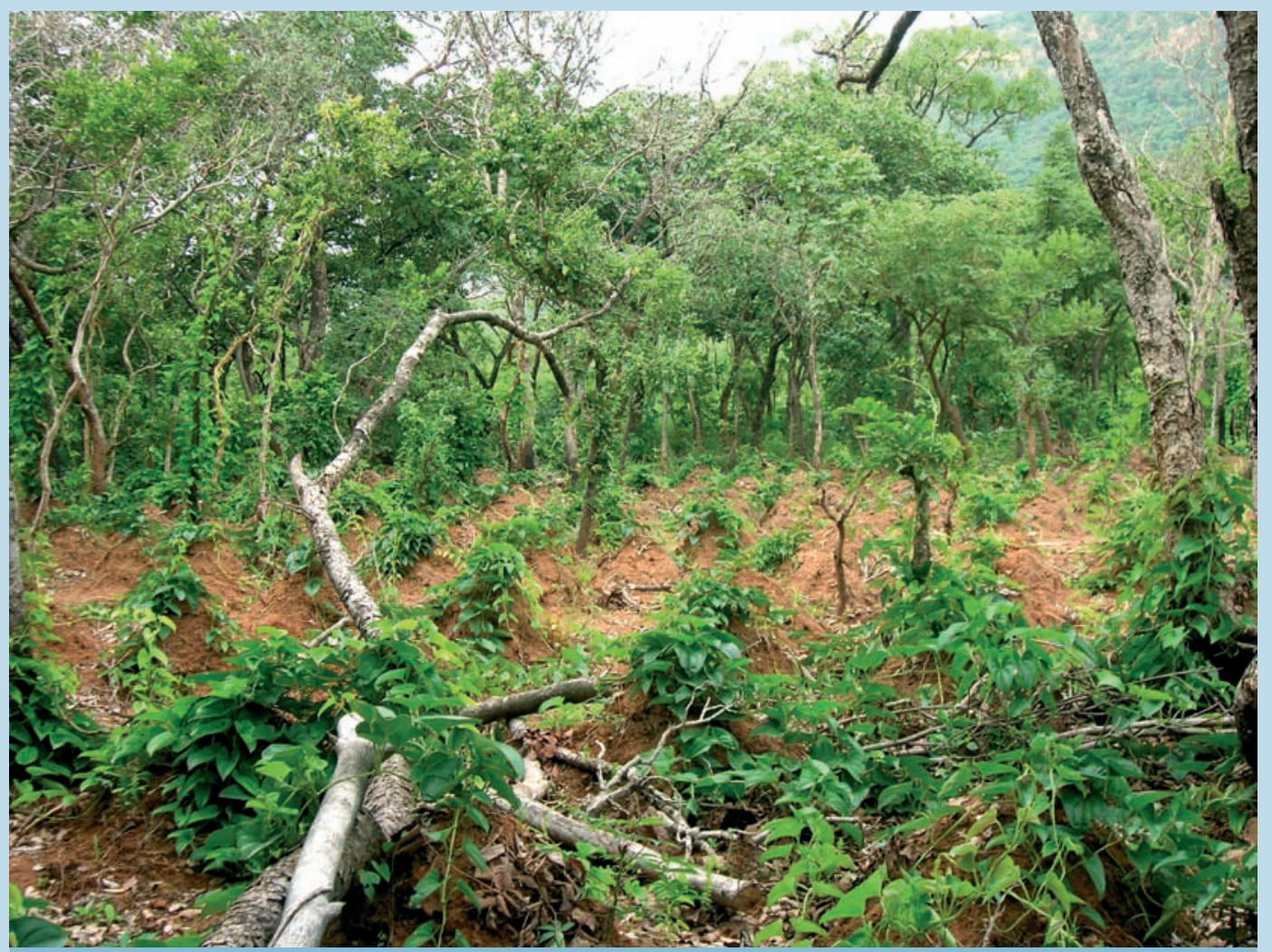

Photo 1.

Forêt claire à Isoberlinia doka et Isoberlinia tomentosa défrichée et mise en culture pour l'igname à Boulowou. Photo M. Dourma. 


\section{RÉSUMÉ}

\section{COMPARAISON DE L'UTILISATION DES RESSOURCES FORESTIÈRES ET DE LA RÉGÉNÉRATION ENTRE DEUX TYPES DE FORÊTS CLAIRES À ISOBERLINIA AU TOGO}

Une étude ethnobotanique sur un échantillon de 135 personnes a permis de définir le rôle de l'homme et les utilisations des ressources végétales au sein des forêts claires à Isoberlinia du domaine soudanien au Nord-Togo. Les caractéristiques structurales et les modes de régénération ont été étudiés sur 28 placeaux de $20 \times 20$ m installés dans six forêts villageoises à accès libre et 28 autres placeaux situés dans la réserve de faune d'Alédjo ayant un statut de protection. La matrice " utilisations $x$ personnes enquêtées " a été soumise à la classification ascendante hiérarchique grâce au logiciel Statistica 6.0. Elle a permis de confirmer le rôle écologique, agricole, économique et vital des ressources végétales à $35 \%$ de dissemblance. Le mode actuel d'exploitation des forêts locales compromet leur pérennité, surtout en l'absence de mesures de sauvegarde. La richesse spécifique est assez semblable dans les deux types de forêts claires. Les indices de Shannon ( 3,77 bits) et d'équitabilité de Piélou $(0,68)$ sont plus élevés dans les forêts protégées que dans celles à accès libre (3,31 bits ; $0,53)$. Ce qui suggère que l'absence d'activités anthropiques favorise une meilleure conservation de la diversité des forêts qui se régénèrent par semis, par rejets de souche et par drageons. La densité (475 tiges/ha), la surface terrière $\left(15,2 \mathrm{~m}^{2} / \mathrm{ha}\right)$, le diamètre moyen $(23,9 \mathrm{~cm})$ et la hauteur moyenne $(15,5 \mathrm{~m})$ des arbres sont plus élevés dans les forêts protégées que dans celles non protégées (300 tiges/ha ; $\left.13,9 \mathrm{~m}^{2} / \mathrm{ha} ; 18,8 \mathrm{~cm} ; 11,9 \mathrm{~m}\right)$.

Mots-clés : forêt claire à Isoberlinia, biodiversité, mode de régénération, protection, Togo.

\section{ABSTRACT}

\section{A COMPARATIVE ANALYSIS OF RESOURCE USE AND REGENERATION IN TWO TYPES OF OPEN ISOBERLINIA WOODLAND IN TOGO}

An ethno-botanical study of a sample population of 135 people was conducted to define the role and patterns of human uses of plant resources in open Isoberlinia woodland in northern Togo's Sudanian region. Structural characteristics and regeneration patterns were investigated in 28 sample plots measuring $20 \times 20 \mathrm{~m}$ established in 6 free-access community forests, and in 28 further plots in the Alédjo wildlife reserve, which is protected. The "uses $x$ people surveyed" matrix, when ranked in ascending order with Statistica 6.0 software, confirmed the ecological, agricultural, economic and subsistence roles of plant resources with $35 \%$ dissemblance. Current patterns of local forest use are jeopardising their survival, especially given the lack of conservation measures. Species richness is fairly similar in the two types of open woodland. Shannon's diversity index (3.77 bits) and Piélou's equitability index (0.68) are higher in protected forests than in those that can be freely used (3.31 bits and 0.53). This suggests that absence of human activities promotes better conservation of forest diversity when trees regenerate by seeding, clump shoots and suckering. Density (475 stems/ha), basal area $\left(15.2 \mathrm{~m}^{2} / \mathrm{ha}\right)$, average diameter $(23.9 \mathrm{~cm})$ and average height $(15.5 \mathrm{~m})$ are higher in protected than unprotected forests (300 stems/ha; $\left.13.9 \mathrm{~m}^{2} / \mathrm{ha} ; 18.8 \mathrm{~cm} ; 11.9 \mathrm{~m}\right)$.

Keywords: open Isoberlinia woodland, biodiversity, regeneration patterns, protection, Togo.

\section{RESUMEN}

\section{COMPARACIÓN DE LA UTILIZACIÓN DE RECURSOS FORESTALES Y DE LA REGENERACIÓN ENTRE DOS TIPOS DE BOSQUES ABIERTOS DE ISOBERLINIA EN TOGO}

Un estudio etnobotánico, realizado sobre una muestra de 135 personas, permitió definir el papel del hombre y los usos de los recursos vegetales en bosques abiertos de Isoberlinia en el dominio climático sudanés del norte de Togo. Se estudiaron las características estructurales y los modos de regeneración en 28 parcelas de $20 \times 20 \mathrm{~m}$ establecidas en 6 bosques comunales de libre acceso y, también, en 28 parcelas situadas en la reserva faunística de Alédjo y que cuentan con protección. A partir de la matriz "usos $x$ personas encuestadas", se efectuó la clasificación ascendente jerárquica mediante el programa Statistica 6.0. Esta clasificación permitió confirmar el papel ecológico, agrícola, económico y vital de los recursos vegetales con un porcentaje de disimilitud del $35 \%$. El actual modo de explotación de los bosques locales compromete su permanencia, sobre todo si no se toman medidas de protección. La riqueza específica es bastante semejante en ambos tipos de bosque abierto. Los índices de biodiversidad de Shannon (3,77 bits) y de equidad de Piélou $(0,68)$ son más elevados en los bosques protegidos que en los de libre acceso (3,31 bits; 0,53). Esto podría sugerir que la ausencia de actividades antrópicas favorece una mejor conservación de la diversidad de los bosques que se regeneran por semillas, brotes de cepa o de raíz. La densidad (475 pies/ha), el área basal $\left(15,2 \mathrm{~m}^{2} / \mathrm{ha}\right)$, el diámetro medio $(23,9 \mathrm{~cm})$ y la altura media $(15,5 \mathrm{~m})$ de los árboles son mayores en los bosques protegidos que en los no protegidos (300 pies/ha; 13,9 m²/ha; $18,8 \mathrm{~cm} ; 11,9 \mathrm{~m})$.

Palabras clave: bosques abiertos de Isoberlinia, biodiversidad, modos de regeneración, protección, Togo. 


\section{Introduction}

L'exploitation des produits forestiers ligneux et non ligneux par les populations locales est courante en forêt tropicale. Les savanes boisées et les forêts claires du domaine soudanien d'Afrique de l'Ouest, formées de peuplements quasi monospécifiques à Isoberlinia doka et I. tomentosa, sont le plus souvent défrichées, notamment à des fins agricoles au Togo (photo 1). Le bois de ces essences est utilisé en menuiserie (bois d'œuvre), en artisanat, comme bois énergie (OURO-DJÉRI, 1994) et comme bois de service, notamment les perches pour la construction. Des vertus médicinales et magico-thérapeutiques sont reconnues pour de nombreuses espèces de ces forêts claires.

En dépit de la valeur socio-économique que représentent ces forêts dans les circuits d'approvisionnement des populations locales, aucune étude n'a été consacrée aux diverses formes d'utilisation de ces ressources ligneuses et non ligneuses. Or, les pressions anthropiques mettent en péril la pérennité des forêts claires à Isoberlinia. Cette situation est inquiétante et ne permet pas d'envisager une gestion durable de ces ressources. Aucun plan d'aménagement ou de conservation ne peut être durable sans l'implication effective des populations locales et de leur savoir-faire traditionnel.

Cet article traite des formes d'utilisation attribuées aux espèces ligneuses par les populations, des savoirs locaux et de l'impact des activités humaines sur le maintien de la diversité au sein des forêts claires à Isoberlinia au Nord-Togo.

\section{Présentation de la zone d'étude}

L'étude a été menée au nord du Togo, entre les longitudes $0^{\circ} 20^{\prime}$ et $1^{\circ} 35^{\prime}$ 'Est et les latitudes $8^{\circ} 15^{\prime}$ et $10^{\circ}$ Nord. Cette zone appartient au centre régional d'endémisme soudanien (WHITE, 1986). Elle inclut les zones écologiques II et III du Togo définies par ERN (1979) (figures 1 et 2). Elle est limitée au nord par les monts Défalé et Kabyè et au sud par les monts Fazao-Malfakassa et Alédjo. Ces deux massifs au relief irrégulier atteignent 400 à 500 m d'altitude et encadrent le plateau de Niamtougou et la plaine de la Binah. Sur le plan géologique, le milieu correspond aux couvertures épimétamorphiques et sédimentaires d'orientation sudouest-nord-est. Les sols ferrugineux et ferrallitiques tropicaux sont dominants. Les cours d'eau qui drainent la zone s'écoulent d'est en ouest pour se jeter dans le fleuve Volta au Ghana. La zone est caractérisée par un climat tropical à régime unimodal (900 à 1400 mm de précipitations

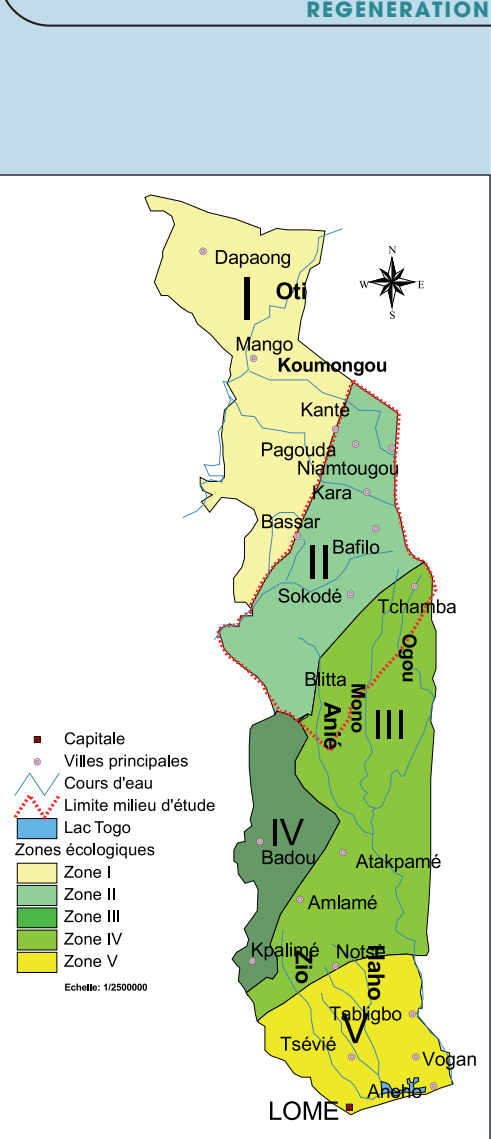

Figure 1.

Zones écologiques du Togo et milieu d'étude (encadré en rouge).

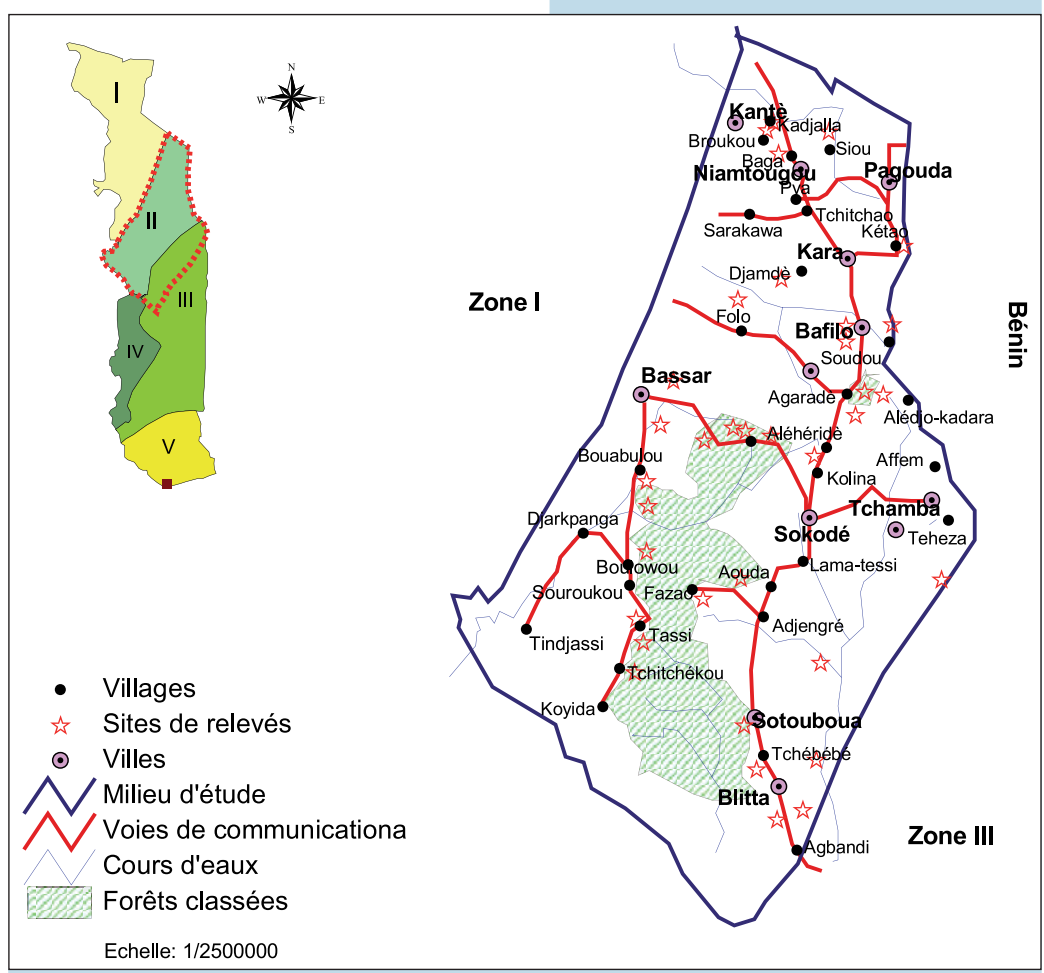

Figure 2.

Distribution des sites d'inventaire forestier et d'enquête. 
par an). Les températures moyennes mensuelles varient de 24,7 à $28,6^{\circ} \mathrm{C}$ avec une humidité relative moyenne de l'air de 40 à $80 \%$.

La population est constituée de groupes ethniques hétérogènes (Cotocolis, Bassar, Kabye, Nawdba,
Lamba, Tamberma, Agnanga et Peuls) qui vivent des revenus de l'agriculture, de l'élevage, du commerce et de l'artisanat. La culture itinérante sur brûlis, l'élevage et la chasse modifient considérablement la flore et la faune.

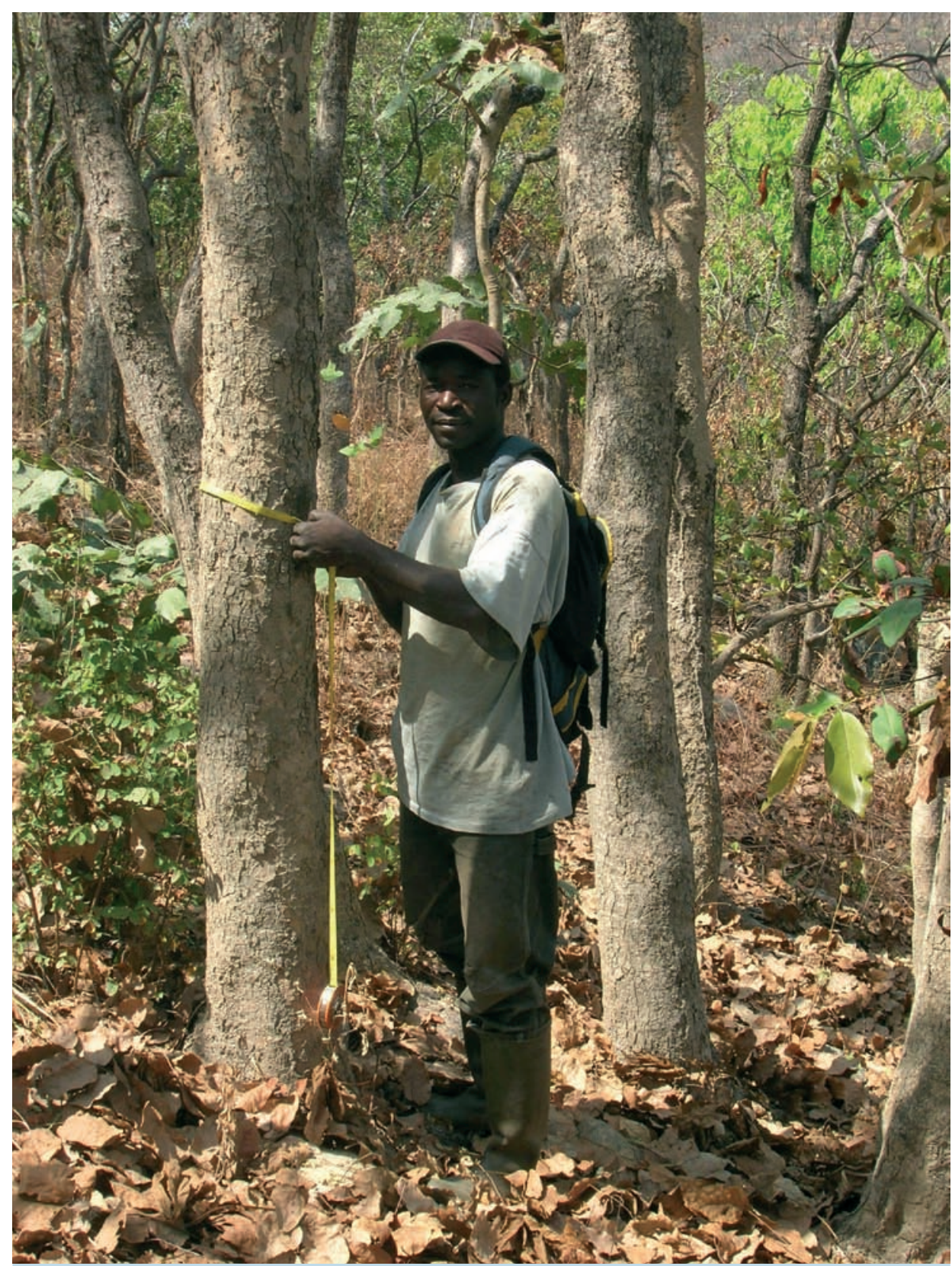

Photo 2.

Inventaire en forêt claire sur /soberlinia à Wiya. Photo M. Dourma.

\section{Matériel et méthodes}

\section{Caractères \\ morphologiques et \\ botaniques des Isoberlinia du Togo}

Isoberlinia doka et I. tomentosa sont des arbres de 10 à $15 \mathrm{~m}$ de haut, pouvant mesurer parfois $20 \mathrm{~m}$, avec un diamètre atteignant 60 à $80 \mathrm{~cm}$ à l'âge adulte. La cime de I. doka est étroite et ouverte, l'écorce du tronc est grise, écailleuse, à tranche rouge clair, alors que I. tomentosa a une cime étalée et dense, son écorce étant grise, écailleuse, à tranche rouge pâle et brune au-dessus (ARBONNIER, 2009). L'appareil végétatif comprend deux à cinq feuilles jugulées à stipules interfoliaires falciformes et caduques. Les feuilles sont alternes, paripennées, de 15 à $30 \mathrm{~cm}$ de long, aux folioles opposées, ovales ou elliptiques, parfois oblongues et tomenteuses chez 1. tomentosa. Les inflorescences sont groupées en panicules. Les fleurs blanches subsessiles sont enveloppées de bractéoles valvaires constituées de cinq sépales linéaires subégaux, de cinq pétales égaux et de 10 à 13 étamines exsertes libres et subégales. Les fruits sont des gousses plates ligneuses de 15 à $30 \mathrm{~cm}$ sur 4 à $7 \mathrm{~cm}$ s'ouvrant en spirales bivalves (GEERLING, 1982).

\section{Phénologie}

La période de feuillaison qui correspond d'ordinaire à la saison sèche est propice pour différencier les deux espèces. Pendant cette phase, I. doka présente des feuilles cireuses et glabres, alors que celles d'l. tomentosa sont grises et veloutées sur les deux faces. Le feuillage devient ensuite peu luisant, rendant difficile la distinction entre les deux espèces. La floraison est étalée de décembre à février et précoce chez I. doka. La fructification couvre les mois de mars à mi-juin. 


\section{Aire de distribution}

Les deux espèces d'/soberlinia se rencontrent précisément dans le domaine soudanien de l'Afrique subsaharienne. En Afrique occidentale, on les retrouve entre $8^{\circ}$ et $13^{\circ}$ de latitude Nord (Mali, Bénin, Togo, Ghana, Côte d'Ivoire, Guinée, Burkina Faso, Niger, Nigeria). En Afrique centrale, elles se retrouvent entre $5^{\circ}$ et $9^{\circ}$ de latitude Nord (Cameroun, Tchad, République centrafricaine, République démocratique du Congo) (WATSON, DALLWITZ, 1993).

\section{Collecte et traitement de données}

Les données sont collectées dans les forêts villageoises des localités de Kolina, Amaïdè, Tabindè, Wiya, Agbang, Broukou où les défrichements agricoles, le pâturage et l'exploitation artisanale du bois contribuent à la dégradation des formations végétales, et dans la forêt claire d'Alédjo, érigée en réserve de faune par l'arrêté $n^{\circ} 411$ du 30 juillet 1930, choisie comme témoin compte tenu de son statut de protection.

Pour connaître les différentes utilisations des espèces ligneuses des forêts claires à Isoberlinia du Togo et l'importance des éventuelles menaces qui pèsent sur ces essences, des enquêtes ethnobotaniques ont été menées auprès des populations locales. Un questionnaire direct à questions ouvertes et semi-structurées a été soumis à 135 personnes (86 hommes, 49 femmes de divers âges). Les questions ont porté sur les formes d'utilisation des produits (ligneux ou non) des espèces exploitées, leurs modalités de reproduction naturelle ainsi que sur l'impact humain sur la biodiversité.

Les données d'enquêtes ont été complétées par des inventaires forestiers sur 28 placeaux de $20 \times 20$ m installés dans les forêts villageoises et 28 autres dans la réserve de faune d'Alédjo en vue de mieux définir le rôle de l'homme dans le devenir des forêts étudiées (figure 2). Dans chaque placeau, tous les arbres dont le diamètre à $1,3 \mathrm{~m}$ était supérieur ou égal à $10 \mathrm{~cm}(\mathrm{~d} \geq 10 \mathrm{~cm})$ ont été inventoriés. Ces diamètres ont été mesurés à l'aide d'un ruban (photo 2) et les hauteurs estimées à la vue. Les souches ont été dénombrées.

Les données collectées ont été saisies dans un tableur et soumises à des traitements statistiques spécifiques. Les diverses utilisations reconnues à la suite de l'enquête ethnobotanique ont été appréhendées par des analyses statistiques multivariées grâce au logiciel Statistica 6.0. Pour ce faire, une matrice " utilisations des espèces $x$ personnes enquêtées » a été élaborée et discriminée à l'aide d'une classification ascendante hiérarchique (Cah). La Cah a permis la classification et l'ordination des enquêtés sui- vant la ressemblance des réponses, afin de mettre en évidence les types d'utilisations des ressources des forêts claires à statut de protection, d'une part, et celles sans aucun statut et subissant une forte emprise anthropique, d'autre part. Les grandeurs de la diversité spécifique, à savoir la richesse spécifique (Rs), l'indice de Shannon ( $H$ ') et l'équitabilité de Piélou (E), ont été calculées. De même, les caractéristiques structurales telles que la densité des tiges $\left(D_{10}\right)$, la surface terrière $(G)$ et le taux de coupe (Tc) ont fait l'objet de calculs. L'analyse de variance a été réalisée ainsi que le test post-hoc de Newman-Keuls entre les caractéristiques structurales et la diversité alpha (MAGURRAN, 1988) en vue d'une comparaison des deux types de forêts claires.

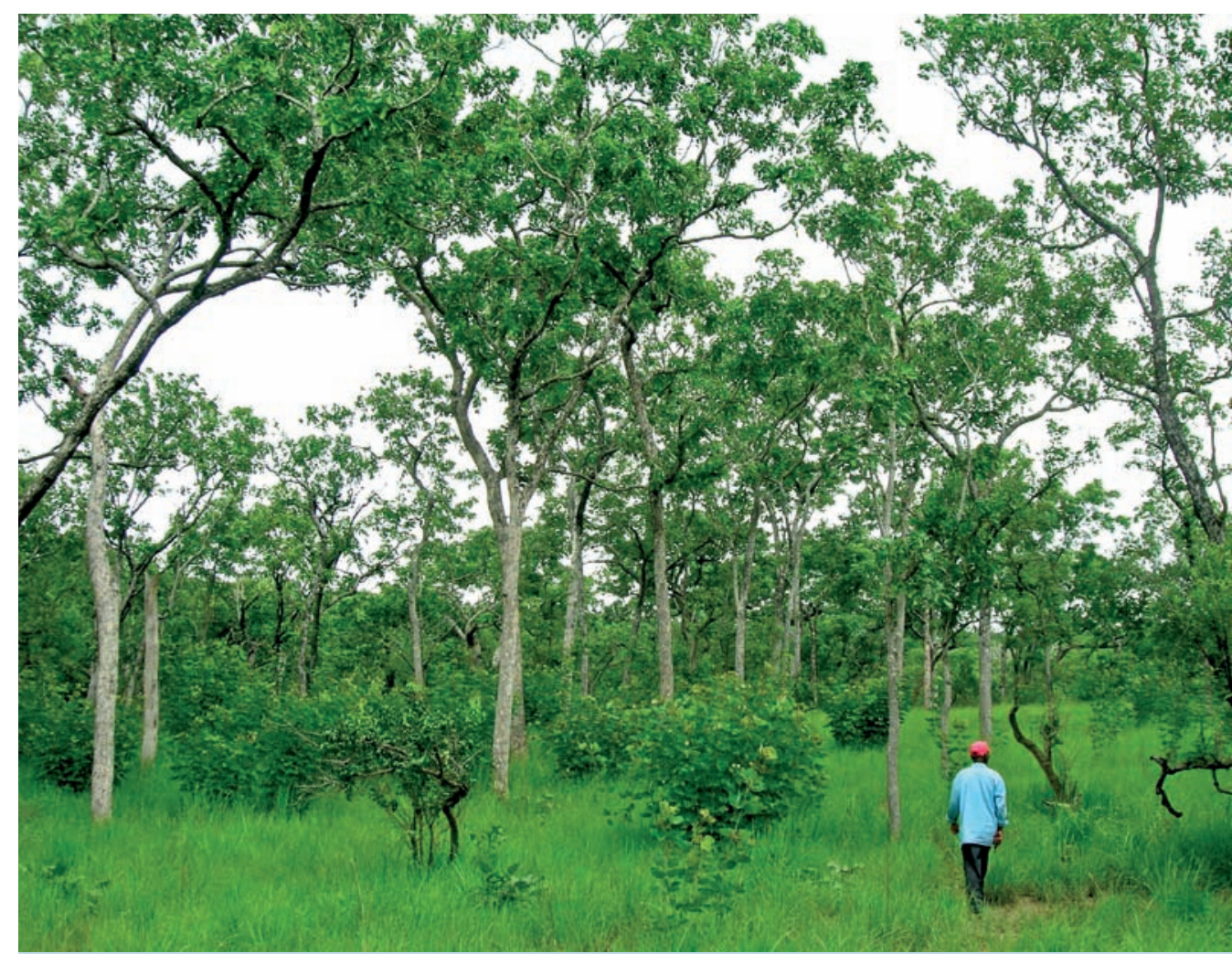

Photo 3.

Forêt claire à Isoberlinia spp.

Photo M. Dourma. 


\section{Résultats}

\section{Utilisations des espèces ligneuses des forêts claires à Isoberlinia et potentiel socio-économique}

Les personnes enquêtées reconnaissent que les deux espèces d'Isoberlinia jouent un rôle important dans leur vie quotidienne. Les populations riveraines attribuent quatre rôles principaux compte tenu de leurs fonctions et utilisations. Au seuil de $35 \%$ de dissemblance, la Cah et l'interprétation discriminent quatre groupes d'enquêtés :

- FU1 qui attribue un rôle écologique aux forêts claires à Isoberlinia ;

- FU2 qui reconnaît le rôle agricole des forêts claires à Isoberlinia ;

- FU3 qui identifie le rôle écono. mique, lié aux revenus de la vente du bois énergie (FU3a), du charbon de bois (FU3b), du bois d'œuvre (FU3c) et du bois de service (FU3d) ;

- FU4 qui concerne le rôle vital, compte tenu de leur fonction alimentaire (FU4a) et de leur importance médicinale (FU4b) dans la vie quotidienne des populations locales (figure 3).

\section{Rôle écologique (FU1)}

Les forêts claires à I. doka affectionnent les substrats édaphiques très profonds à texture sablo-argileuse (photo 3). Par contre, celles à I. tomentosa se localisent sur les sols peu profonds à gravillons et affleurements rocheux. En général, le substrat édaphique influence la répartition des espèces d' $\%$ doka qui préfèrent les sols riches en humus, par opposition aux espèces d'l. tomentosa qui affectionnent les sols relativement pauvres. Les sites à I. tomentosa sont marqués par une érosion de l'horizon superficiel du sol, rendant ces sols impropres aux cultures. Cela explique pourquoi les sols à $I$. doka sont très prisés pour l'exploitation agricole. L'importance du système radiculaire latéral, couplée à l'aptitude naturelle à drageonner des deux espèces (Dourma et al., 2006), permet de conserver la structure du sol sur les terrains à forte pente, et de prévenir l'érosion hydrique et éolienne des sols. En plus de ce rôle écologique éminent, ces forêts représentent des aires de parcours et un ombrage pour le bétail, ainsi qu'un habitat de prédilection pour la faune sauvage.

\section{Rôle agricole (FU2)}

Dans les parcelles mises en culture, les jeunes baliveaux conservés et les troncs d'arbres écorcés et tués sur pied servent de tuteurs pour la culture de l'igname. l. doka caractérise les sols riches en litière et en matière organique, alors que $I$. tomentosa affectionne les sols relativement pauvres. Ces sols à horizon superficiel, très érodés, sont impropres aux cultures. Les racines pivotantes et obliques d'Isoberlinia permettent une exploitation des eaux souterraines profondes et des remontées favorables aux cultures. Cela explique pourquoi les sols à $I$. doka sont très prisés par les agriculteurs. Les feuilles tombées constituent un engrais vert pour les cultures.

\section{Rôle économique (FU3)}

Les revenus liés à la vente du bois énergie (FU3a), du charbon de bois (FU3b), du bois d'œuvre (FU3C) et du bois de service (FU3d) améliorent de façon indéniable le niveau de vie de certains ménages.

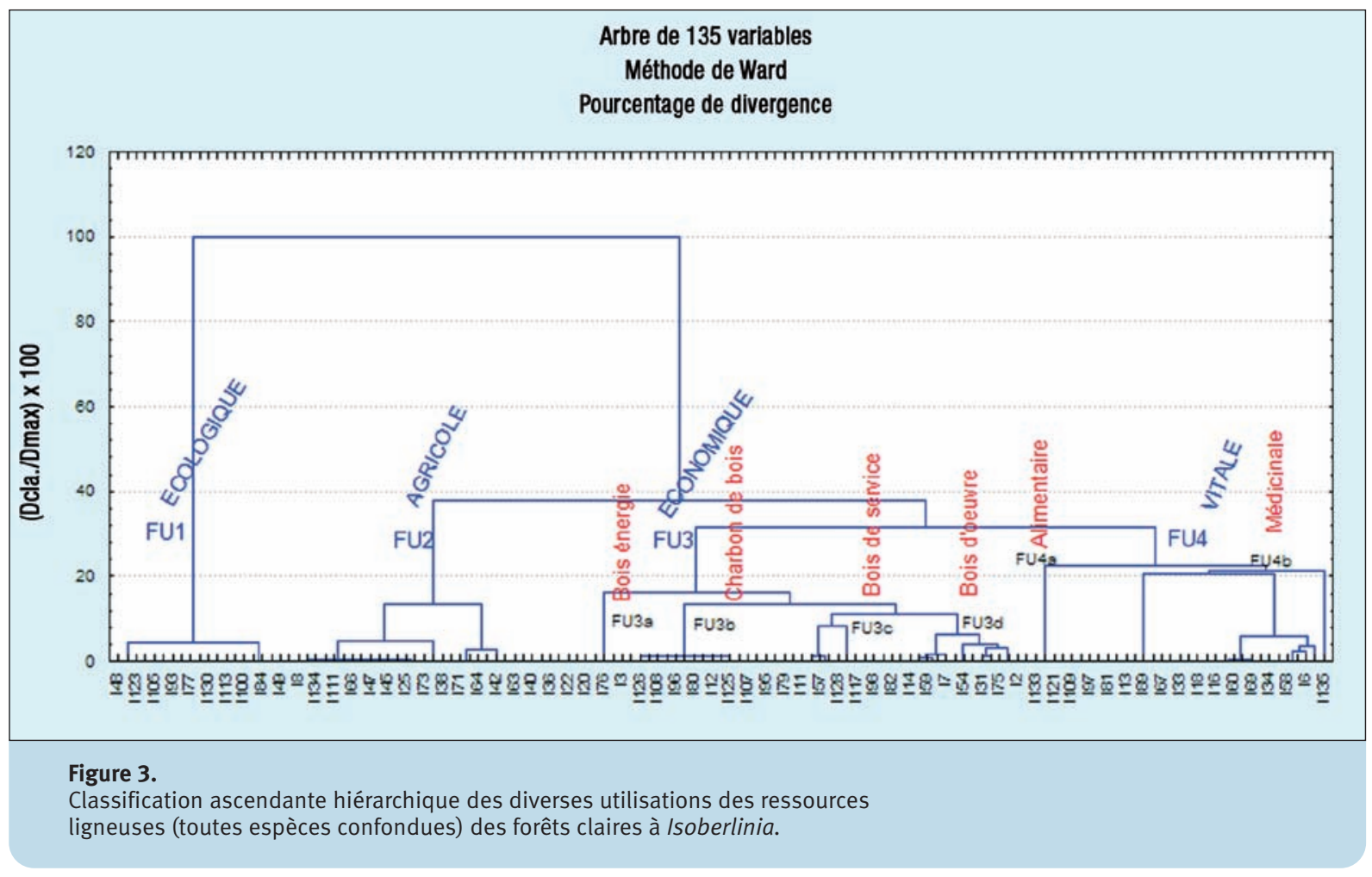




\section{Bois énergie (FU3a)}

Le bois des espèces ligneuses des forêts claires à Isoberlinia est apprécié comme source d'énergie domestique traditionnelle par les ménages et pour la commercialisation (photo 4). La vente du bois énergie relève des activités des femmes, surtout celles qui sont mariées (figure 4). Dans cette région, les femmes ont un rôle important dans la gestion des dépenses du ménage. Le fagot de $10 \mathrm{~kg}$ est vendu 200 francs $\mathrm{Cfa}(0,3 €)$ et les recettes hebdomadaires sont évaluées à 2000 francs Cfa (environ $3 €)$. Le bois vendu est dirigé vers les villes voisines (Sokodé, Kara), voire parfois Lomé. Des quantités non négligeables de bois énergie et de charbon de bois sont convoyées à l'extérieur du pays, vers Ouagadougou.

\section{Charbon de bois (FU3b)}

La fabrication du charbon de bois est relativement récente; elle connaît aujourd'hui une ampleur considérable (photo 5). Le charbon peut être fabriqué uniquement à base d'Isoberlinia ou en mélange avec d'autres espèces : les charbonniers abattent à la tronçonneuse des pieds de Isoberlinia, Anogeissus leiocarpus, Pterocarpus erinaceus, etc. afin de produire du charbon de bois pour les commerçantes venues de la ville. Le sac de $25 \mathrm{~kg}$ est vendu 1000 francs $\mathrm{Cfa}(1,5 €)$ sur place et plus s'il est vendu en ville.

\section{Bois d'œuvre (FU3c)}

Les forêts locales sont pauvres en bois d'œuvre de valeur. Cette situation conduit les populations à exploiter Isoberlinia comme bois d'œuvre (photos 6). Les gros arbres sont débités en planches, chevrons et madriers par des exploitants allochtones et ensuite convoyés vers les villes pour la vente en vue de leur utilisation en menuiserie et dans la construction. Les planches sont vendues entre 2000 et 2500 francs Cfa ( 3 et $3,8 €$ ), tandis que les chevrons valent 1800 francs $\mathrm{Cfa}(2,75 €)$.

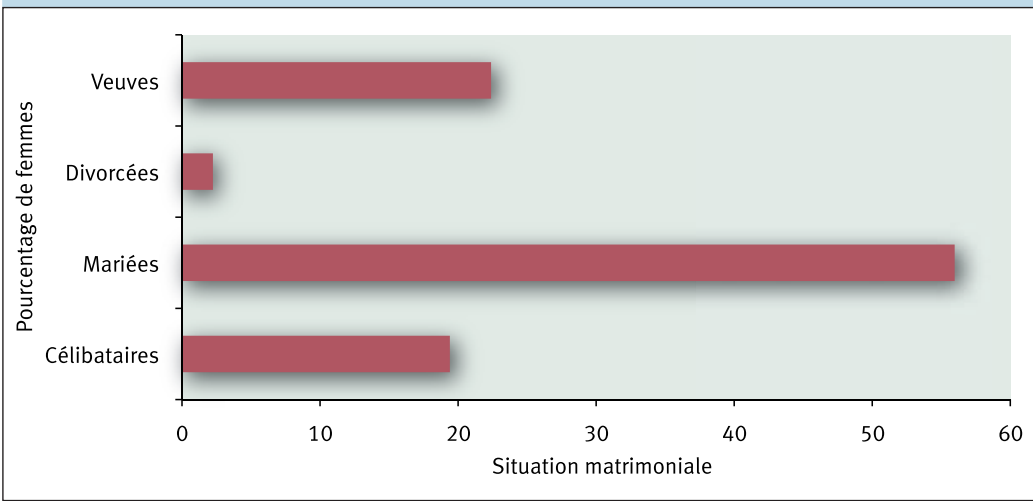

Figure 4.

Répartition des vendeuses de bois énergie en fonction de leur statut matrimonial.

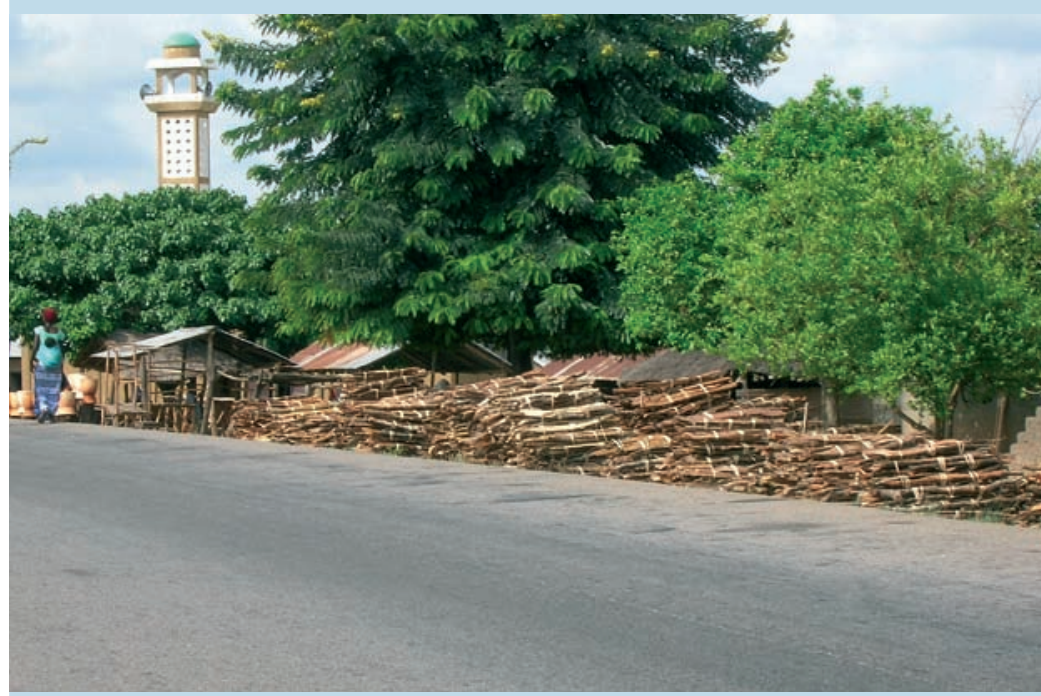

Photo 4.

Vente de bois de feu le long de la route à Azanadè.

Photo M. Dourma.

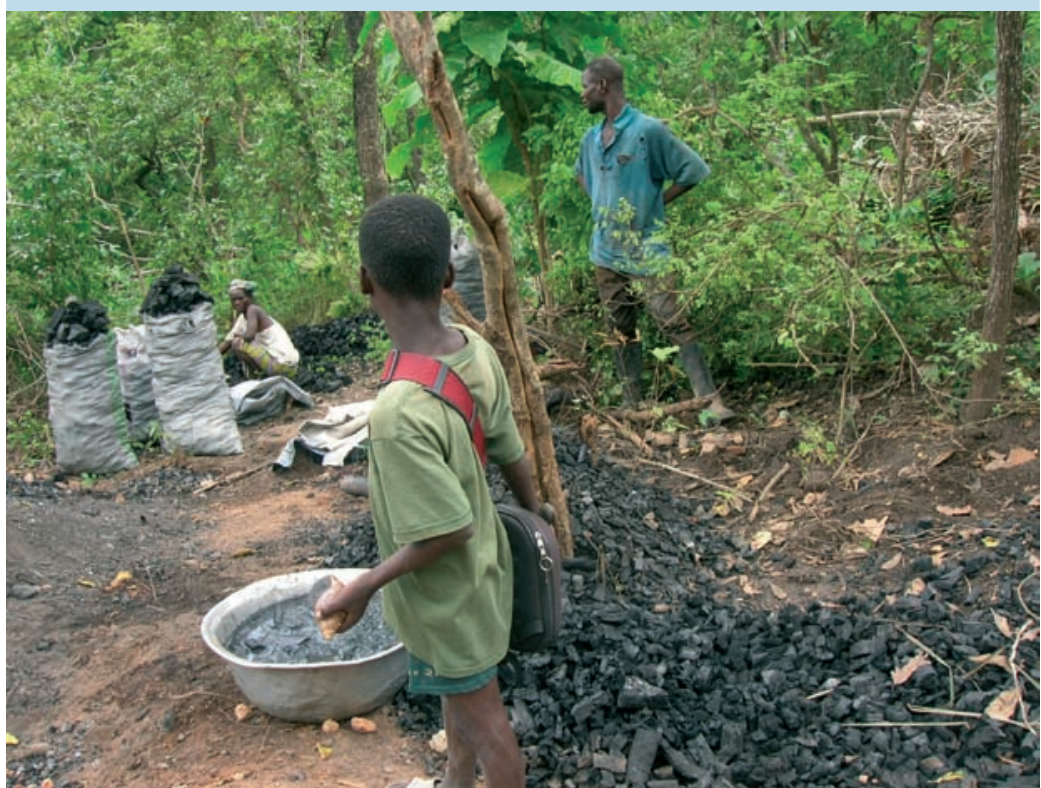

Photo 5.

Production de charbon de bois à base d'Isoberlinia en forêt à Kolina. Photo M. Dourma. 


\section{Bois de service (FU3d)}

De nombreuses perches d'Isoberlinia sont utilisées pour la construction des maisons. Les jeunes brins sont prélevés à cet effet et vendus le long de la route (photos 7). Les perches sont également convoyées vers les villes (Sokodé, Atakpamé, Bafilo, Kara, Dapaong, etc.) et servent d'échafaudage. D'autres sont utilisées pour les charpentes des hangars et comme tables d'étalage de marchandises et sièges dans les marchés locaux. Ces perches constituent également des étagères démontables sur les remorques de camions pour le transport de tomates, de fruits et du petit bétail. La récolte se fait parfois sur commande du client, si la demande est élevée. Les prix de vente varient de 150 à 300 francs Cfa $(0,22$ à $0,45 €)$ en fonction de la quantité. Le bois d'Isoberlinia sert aussi à fabriquer des mortiers, des pilons, divers outils et des cales pour camions. Cette dernière activité, génératrice de revenus non négligeables, est souvent le fait des jeunes (écoliers ou non) ; la cale est vendue entre 800 et 1000 francs Cfa (1,2 à $1,5 €)$ le long des routes. Un tronc d'arbre adulte de $15 \mathrm{~m}$ de haut produit environ 20 cales, ce qui génère une somme de 16000 à 20000 francs Cfa (24,4 à 30,5 €).

En plus des divers produits ligneux cités ci-dessus, les feuilles d' $\%$ doka servent à emballer les condiments dans les marchés et à la cuisson des mets locaux. En artisanat, les gousses des fruits sont utilisées pour polir les pots en terre cuite lors de leur fabrication.

La commercialisation de tous ces produits procure des revenus non négligeables à la plupart des foyers. Les espèces utilisées dans ces forêts claires sont, entre autres, Isoberlinia doka, I. tomentosa, Pericopsis laxiflora, Daniellia oliveri, Lannea acida, Monotes kerstingii, Parinari curatellifolia, Entada africana, Terminalia glaucescens, Pterocarpus erinaceus, Burkea africana, Uapaca togoensis. 


\section{Rôle vital (FU4)}

Les forêts à Isoberlinia constituent un réservoir de ressources végétales essentielles à rôle médicinal et alimentaire pour les populations locales.

\section{Rôle médicinal (FU4a)}

Les différents organes d'Isoberlinia sont utilisés seuls ou en association avec d'autres plantes à des fins médicinales. L'écorce du tronc et des racines est utilisée en infusion pour guérir des plaies incurables ; elle permet aussi de traiter les maux de cœur, les hépatites, les vers intestinaux, les abcès, les convulsions. Les racines superficielles sont très recherchées à des fins magico-thérapeutiques : celles d'l. doka sont utilisées pour confectionner des talismans de protection contre les malédictions et celles d'l. tomentosa pour confectionner des contre-poisons. L'enquête a également fait ressortir que Nauclea latifolia, Vitellaria paradoxa, Pavetta crassipes, Securidaca longepedunculata, Prosopis africana sont utilisées dans la pharmacopée traditionnelle.

\section{Rôle alimentaire (FU4b)}

Les abeilles butinent les fleurs de nombreuses essences des forêts claires et érigent leurs ruches sur les espèces des genres Lannea, Vitellaria, Parkia, Vitex, etc. Nombreuses sont les espèces qui sont également sollicitées pour leurs fruits. Il s'agit, entre autres, de Nauclea latifolia, Vitellaria paradoxa, Detarium microcarpa, Parinari curatellifolia, Parkia biglobosa, Prosopis africana. Les graines des deux espèces d'/soberlinia constituent aussi une source alimentaire très appréciée par la faune sauvage et les insectes. Enfin, de nombreuses espèces de champignons comestibles sont récoltées dans les forêts claires à Isoberlinia.
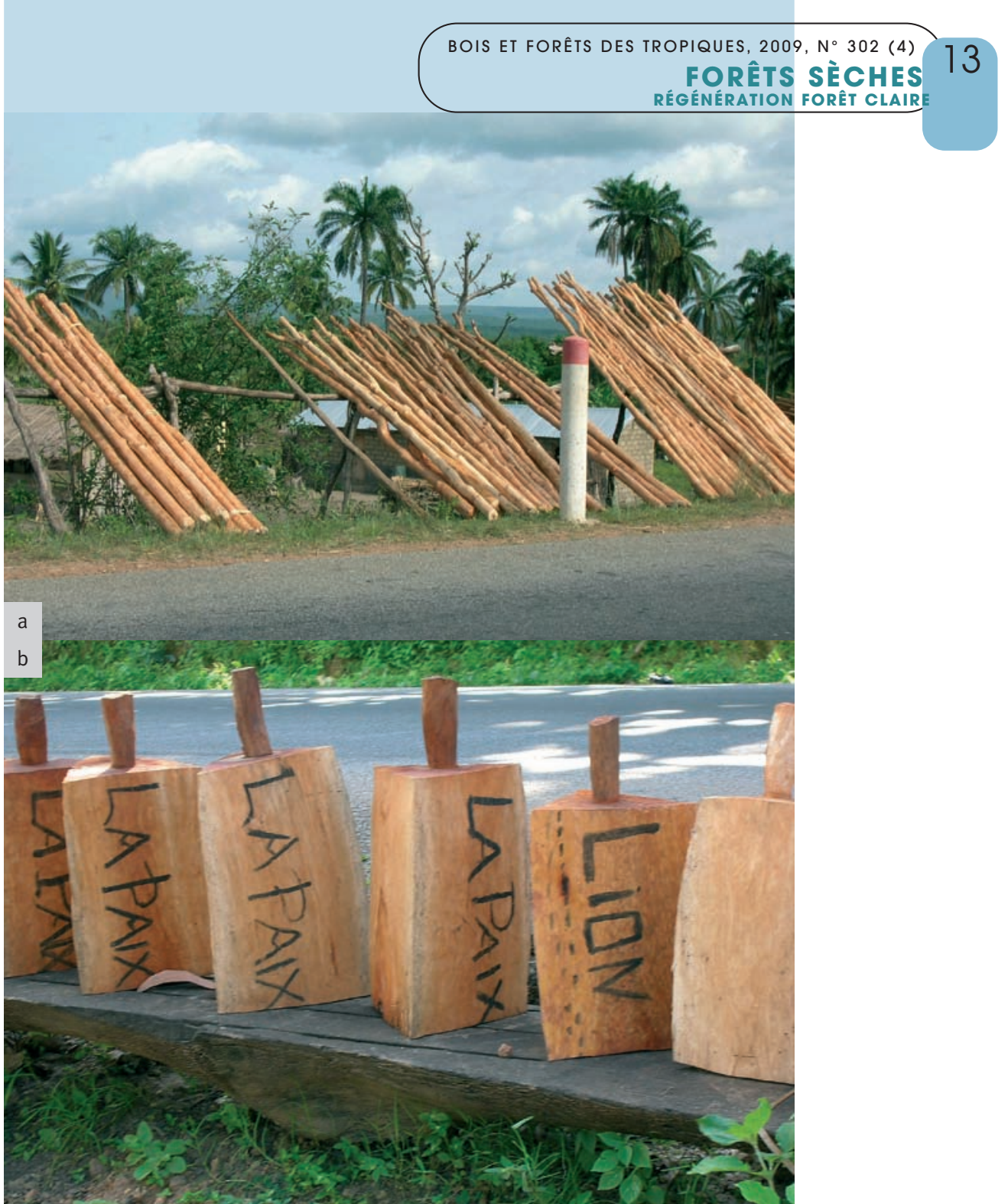

Photos 7.

Vente de perches pour échafaudages (a) et de cales pour camions (b) produites à base du bois d'Isoberlinia à Amaïdè.

Photos M. Dourma.

\section{Savoirs locaux relatifs aux modes de régénération des espèces ligneuses}

Les données recueillies par ces enquêtes montrent que la régénération dans ces forêts claires s'obtient soit par semis, drageons et rejets de souche en fonction des espèces. En effet, nombreuses sont les espèces ligneuses locales qui résultent de la germination de graines tombées de l'arbre à maturité. Cependant, selon certains enquêtés, la coupe de l'arbre à des périodes favorables de l'année favorise l'apparition de nombreux drageons sur les racines traçantes (photo 8 ) et de rejets de souche. Il en va de même après brû- lage de la base des troncs ou à la suite du déracinement partiel du pied par chablis (photo 9). Les drageons apparaissent sur le système racinaire superficiel et proche de la surface du sol. Les rejets de souche s'observent fréquemment sur les troncs d'arbres coupés ou blessés. Les racines les plus aptes à drageonner ont 2,5 à $5 \mathrm{~cm}$ de diamètre. Lorsque des racines sont coupées (lors des labours ou après un chablis), des drageons autonomes se remarquent sur les parties séparées de la racinemère. Les résultats des enquêtes montrent que toutes les racines n'ont pas la même faculté à drageonner en tous ces points. Les drageons sont plus nombreux sous le houppier de l'arbre qu'en 


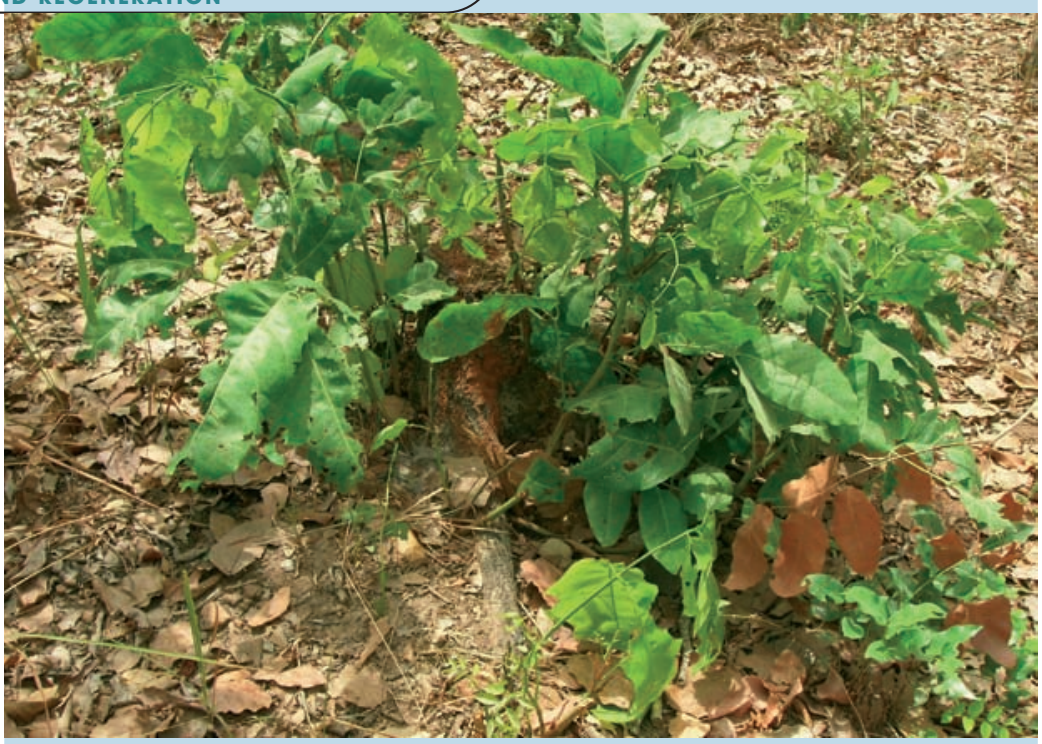

Photo 8.

Régénération par voie végétative (rejets et drageons) d'/soberlinia suite à la coupe du tronc.

Photo M. Dourma.

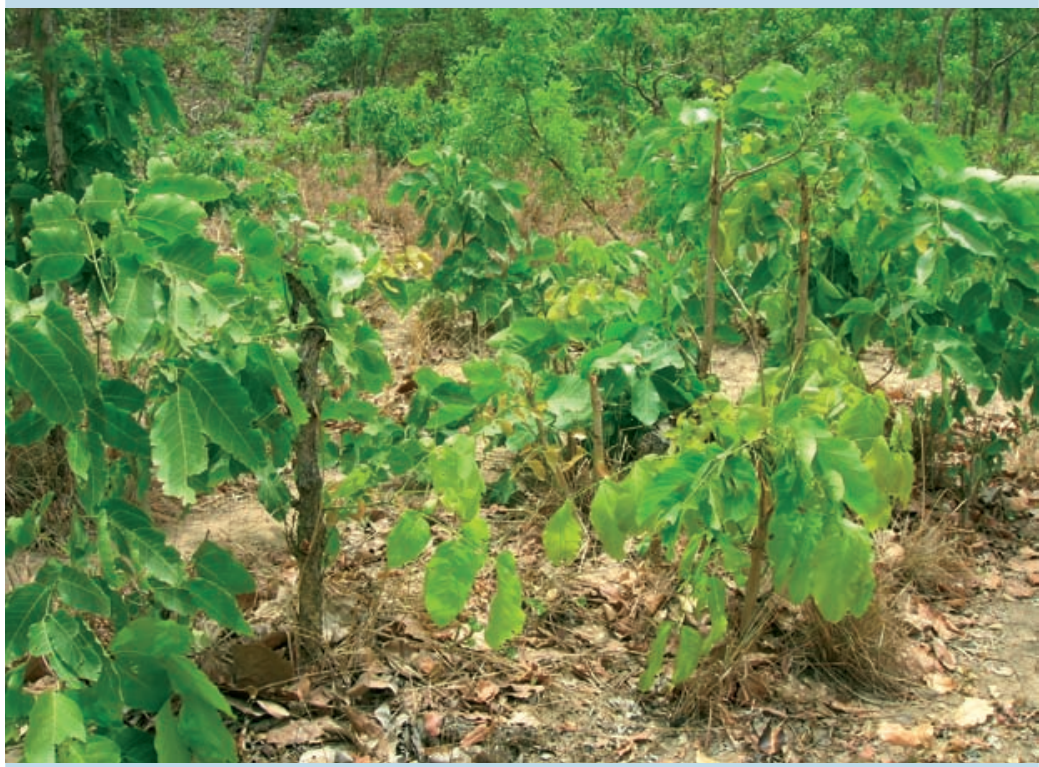

Photo 9.

Stimulation de la multiplication végétative d'/soberlinia spp. par les feux de brousse.

Photo M. Dourma.

dehors de celui-ci. Les arbres adultes drageonnent mieux que les jeunes. Certaines personnes interrogées signalent que les drageons apparaissent en fin de saison sèche chaude et à l'arrivée des pluies (avril-mai) sur les faces supérieure ou latérale des racines (traumatisées ou saines).

Le diagramme rang-fréquence absolu confirme que I. doka, I. tomentosa, Daniellia oliveri, Milicia excelsa, Diospyros mespiliformis drageonnent abondamment dans les parcelles cul-

\section{Gestion locale des ressources naturelles}

En ce qui concerne l'utilisation des ressources naturelles des forêts claires, le constat est très alarmant dans les formations végétales de la zone par manque de gestion et nonrespect de la réglementation. Cette situation entraînera à long terme la disparition des essences locales et surtout des Isoberlinia, si aucune mesure de sauvegarde n'est envisagée. Les défrichements des forêts locales à des fins agricoles et l'exploitation abusive et frauduleuse des espèces végétales sont fréquents dans ces formations végétales. Les réserves de Fazao-Malfakassa et d'Alédjo sont envahies par les populations autochtones, entraînant une dégradation de leur biodiversité.

Ces réserves à statut de protection formelle sont transformées en lieu de parcours et de transit de bétail des Peuls transhumants. Des stations de repos de troupeaux de bœufs ont été observées dans la réserve de faune d'Alédjo lors des prospections. Face à cette menace, les services en charge du secteur forestier sont inefficaces et inactifs. Du point de vue écologique, l'élevage des bovins a un effet destructeur facilement appréciable sur le couvert végétal. Les feux de brousse intentionnels sont provoqués pour régénérer les pâturages. En saison sèche, l'émondage excessif d'arbres fourragers observé vers Kolinia et Amaoudè-Mô est aussi l'une des causes de dégradation des forêts claires à Isoberlinia.

En revanche, certaines pratiques animistes observées favorisent parfois la conservation de la biodiversité des forêts. Des peuplements à Isoberlinia sont transformés en cimetières par les populations locales. En effet, chez les Tem, Agnanga, Lamba et Tamberma, les tombes sont creusées souvent sous couvert arboré. Les populations rapportent que l'ombrage du feuillage protégerait l'esprit du défunt d'éventuelles intempéries. Ainsi, pour témoigner leur respect aux esprits 
des défunts, les espèces de ces forêts-cimetières sont délibérément préservées par les populations locales. Plus au sud de la zone d'étude, une forêt villageoise sacrée à dominance d'l. doka, recouvrant une colline, est préservée par les Agnanga d'Agbandi. Selon les autochtones, tout prélèvement dans cette réserve sacrée inciterait les esprits maléfiques à incarner le malheur sur le village. Pour éviter des conséquences dramatiques, les autochtones veillent sur cette forêt. Tout contrevenant aux principes établis, une fois surpris, a l'obligation d'offrir des sacrifices aux divinités en guise de réparation du préjudice

Espèces

Securidaca longipedonculata Ficus glumosa Albizia zygia Vitex doniana Parinari curatellifolia Afzelia africana

Annona senegalensis Blighia sapida

Prosopis africana

Nauclea latifolia

Millettia thonningii

Anogeissus leiocarpus

Terminalia glauscescens

Vitellaria paradoxa

Anacardium occidentale

Pterocarpus erinaceus

Lannea acida

Adansonia

Uapaca togoensis

Parkia biglobosa

Pentadesma butyracea

Diospyros mespiliformis

Milicia excelsa

Danielia oliveri

Isoberlinia tomentosa

Isoberlinia doka

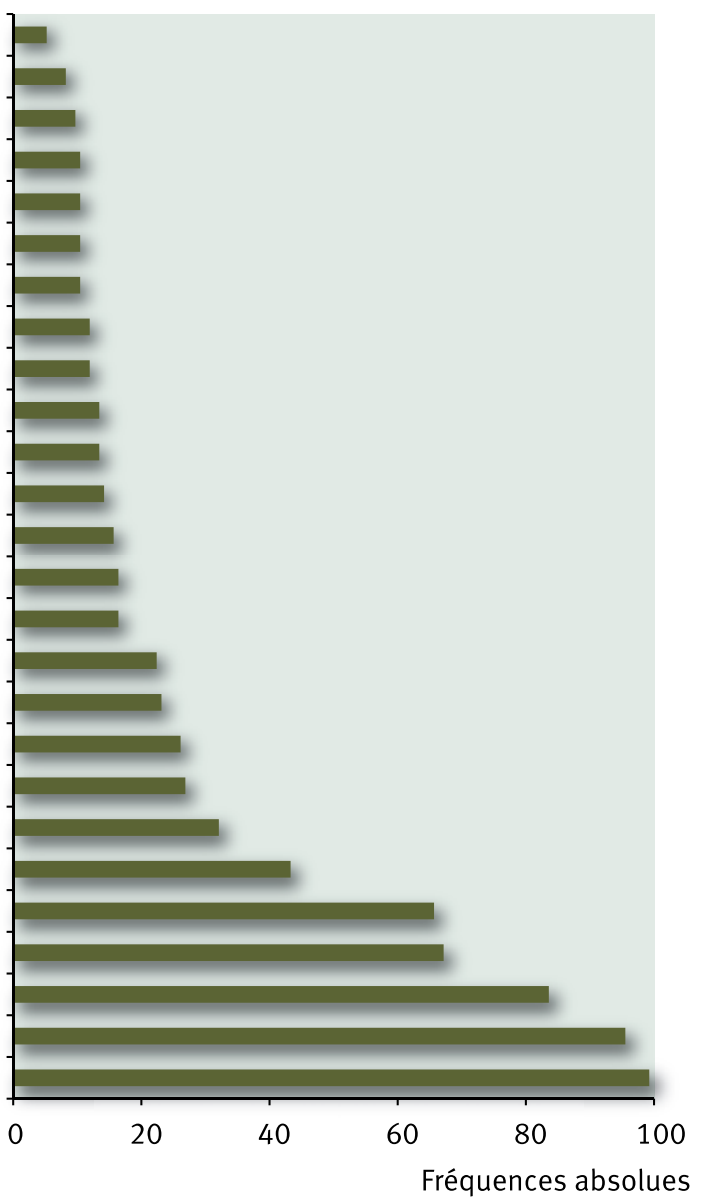

Fréquences absolues

Figure 5.

Diagramme rang-fréquence absolu des espèces ligneuses qui se régénérent par drageonnage. causé. Les rites traditionnels sont l'apanage d'un prêtre coutumier qui a libre accès au lieu du sacrifice, situé au cœur de la forêt.

\section{Impact de l'exploitation sur la biodiversité}

L'enquête révèle que les populations sont conscientes que le rythme d'exploitation des forêts claires compromet leur pérennité. En dehors de la législation existante, aucune mesure de sauvegarde n'a été mise au point pour protéger la biodiversité.

\section{Diversité spécifique}

La diversité spécifique est élevée dans les forêts protégées, mais le nombre de tiges adultes est négligeable dans les forêts non protégées : - La richesse spécifique des forêts protégées (31 espèces ligneuses) est assez similaire à celle des forêts non protégées (28 espèces) ; il n’y a pas de différence significative suivant le test de Newman-Keuls (au seuil de $5 \%, P=0,681)$. Dans ces forêts claires, les espèces d' $/$. doka et d'l. tomentosa sont souvent en association avec d'autres espèces ligneuses. Les plus abondantes sont Pterocarpus erinaceus, Daniellia oliveri, Afzelia africana, Vitellaria paradoxa, Parkia biglobosa, Burkea africana, etc.

- Les indices de Shannon et d'équitabilité de Piélou sont plus élevés dans les zones à statut de protection par rapport aux zones à accès libre. La différence entre les valeurs de l'indice de Shannon (tableau II) est significative entre les deux types de forêts claires au seuil de $2 \%\left(t_{\text {th }}=2,33 \mathrm{au}\right.$ seuil de $2 \%>t_{\text {obs }}=2,48$ avec $d d l$ =641). En effet, la moindre fréquence d'activités anthropiques (agriculture, pâturage, exploitation de bois) dans les zones protégées favorise une meilleure conservation de la diversité biologique des écosystèmes. Le taux d'exploitation dans les forêts non protégées est nettement supérieur à celui des forêts protégées. Ainsi, une exploitation anarchique sans plan d'aménagement durable entraîne une érosion quantitative et qualitative de la diversité des ressources ligneuses. 
Tableau I.

Espèces ligneuses qui drageonnent.

\begin{tabular}{|c|c|c|c|c|}
\hline $\mathrm{N}^{\circ}$ & Famille & Nom scientifique & Nom local & Formation \\
\hline \multirow[t]{2}{*}{1} & \multirow[t]{2}{*}{ Anacardiaceae } & Anacardium occidentale & Atchan, atsan (Tem, Kabyè) & Savane ou bordure de forêt \\
\hline & & Lannea acida & Kongoulou (Tem) & Savane \\
\hline 2 & Annonaceae & Annona senegalensis & Tchoutchoudè (Tem, Kabyè) & Savane \\
\hline 3 & Bombacaceae & Adansonia digitata & Telou (Tem, Kabyè) & Savane \\
\hline \multirow[t]{4}{*}{4} & \multirow[t]{4}{*}{ Caesalpiniceae } & Afzelia africana & Welou (Tem) & Savane/forêt claire \\
\hline & & Danielia & Tchèlè (Tem) & Savane claire \\
\hline & & Isoberlinia doka & Tawrè koufolmou (Tem) & Forêt claire \\
\hline & & Isoberlinia tomentosa & Tawrè kissèmou (Tem) & Forêt claire \\
\hline 5 & Chrysobalanaceae & Parinari curatellifolia & Mamarou (Tem) & Savane \\
\hline \multirow[t]{2}{*}{6} & \multirow[t]{2}{*}{ Combretaceae } & Anogeissus leiocarpus & Tchininga (Tem) & Savane/forêt claire \\
\hline & & Terminalia & Sowoo (Tem) & Savane \\
\hline 7 & Ebenaceae & Diospyros mespiliformis & Tigbado (Tem) & Savane/forêt claire \\
\hline 8 & Euphorbiaceae & Uapaca togoensis & Kidjelim (Tem) & Forêt claire \\
\hline \multirow[t]{2}{*}{9} & \multirow[t]{2}{*}{ Fabaceae } & Millettia thonningii & $?$ & Savane/forêt claire \\
\hline & & Pterocarpus erinaceus & Temou (Tem) & Savane \\
\hline 10 & Guttiferae & Pentadesma butyracea & Agbototewou (Tem) & Forêt claire \\
\hline \multirow[t]{3}{*}{11} & \multirow[t]{3}{*}{ Mimosaceae } & Albizia zygia & Tchinglii-kpalou (Tem) & Forêt claire \\
\hline & & Prosopis africana & Kpalou (Tem) & Savane \\
\hline & & Parkia biglobosa & Solo (Tem) & Savane \\
\hline \multirow[t]{2}{*}{12} & \multirow[t]{2}{*}{ Moraceae } & Ficus glumosa & Fourou (Tem) & Savane \\
\hline & & Milicia excelsa & Sere (Tem) & Forêt claire \\
\hline 13 & Polygalaceae & Securidaca longipedunculata & Fosè (Tem) & Savane \\
\hline 14 & Rubiaceae & Nauclea latifolia & $?$ & Savane \\
\hline 15 & Sapindaceae & Blighia sapida & Fourmou (Tem) & Forêt claire \\
\hline 16 & Sapotaceae & Vitellaria paradoxa & Somou (Tem) & Savane \\
\hline 17 & Verbenaceae & Vitex doniana & Tchimarou (Tem) & Savane \\
\hline
\end{tabular}

Tableau II.

Diversité spécifique et caractéristiques structurales des forêts claires suivant le statut de protection.

\begin{tabular}{|c|c|c|c|c|c|c|c|c|}
\hline $\begin{array}{l}\text { Statut du site } \\
\text { d'étude }\end{array}$ & $\begin{array}{c}\text { Richesse } \\
\text { spécifique totale }\end{array}$ & $\begin{array}{c}\text { Indice de } \\
\text { Shannon (bits) }\end{array}$ & $\begin{array}{c}\text { Équitabilité } \\
\text { de Piélou }\end{array}$ & $\begin{array}{c}\text { Hauteur } \\
\text { moyenne }(\mathrm{m})\end{array}$ & $\begin{array}{l}\text { Diamètre } \\
\text { moyen }(\mathrm{cm})\end{array}$ & $\begin{array}{l}\text { Densité } \\
\text { (tiges/ha) }\end{array}$ & $\begin{array}{c}\text { Surface } \\
\text { terrière }\left(\mathrm{m}^{2} / \mathrm{ha}\right)\end{array}$ & $\begin{array}{l}\text { Taux de } \\
\text { coupe (\%) }\end{array}$ \\
\hline $\begin{array}{l}\text { Forêts claires } \\
\text { protégées }\end{array}$ & $31 \pm 9$ & 3,77 & $0,68 \pm 0,01$ & $15,5 \pm 3,7$ & $23,9 \pm 10,7$ & $475 \pm 98$ & $15,2 \pm 6,9$ & $4,6 \pm 1,4$ \\
\hline $\begin{array}{l}\text { Forêts claires } \\
\text { non protégées }\end{array}$ & $28 \pm 12$ & 3,31 & $0,53 \pm 0,12$ & $11,9 \pm 1,7$ & $18,8 \pm 2,8$ & $300 \pm 42$ & $13,9 \pm 7,4$ & $37,5 \pm 10,3$ \\
\hline
\end{tabular}




\section{Caractéristiques structurales}

La densité des tiges est plus élevée dans les forêts claires protégées que dans celles à accès libre au seuil de $5 \%(P=0,025 ; F=0,039)$. Les valeurs des hauteurs et des diamètres moyens des arbres évoluent dans le même sens avec des différences significatives (respectivement pour $\mathrm{P}=0,0309 ; \mathrm{F}=0,123$ et $P=0,029 ; F=0,312)$. Pour la surface terrière, aucune différence significative n'est enregistrée au seuil de $5 \%$ $(P=0,154 ; F=0,045)$.

Les effectifs des arbres (toutes espèces confondues) regroupés par classes de diamètre sont plus élevés dans les forêts protégées que dans celles à accès libre, sauf pour la classe correspondant à un diamètre supérieur à $50 \mathrm{~cm}$ (figure 6). Cette situation s'explique par le statut de conservation accordé à certains ligneux pour leurs rôles multi-usages (Parkia, Vitellaria, etc.) dans les parcelles cultivées. Dans la forêt protégée, le nombre des tiges décroît assez régulièrement au fur et à mesure que le diamètre augmente, contrairement au cas observé en forêt non protégée. Dans les forêts non protégées, on note aux deux extrémités de la courbe plus d'arbres de très faibles diamètres (semis, drageons) et de très grands diamètres (arbres multi-usages). Cette distribution des tiges des arbres par classes de diamètre est mieux ajustée à une fonction exponentielle aussi bien pour les forêts à statut de protection $\left(y=494,42 e^{-0,43 x} ; R^{2}=0,97\right)$ que celles sans statut de protection $\left(y=71,74 e^{-0,31 x} ; R^{2}=0,47\right)$.

En forêt protégée, les effectifs des arbres par classes de hauteur sont supérieurs à ceux observés en forêt non protégée. De plus, les arbres de hauteur intermédiaire sont plus représentés pour les deux types de forêts. Cette distribution des tiges par classes de hauteur est assimilable à une structure dissymétrique, que ce soit dans les forêts claires pro-

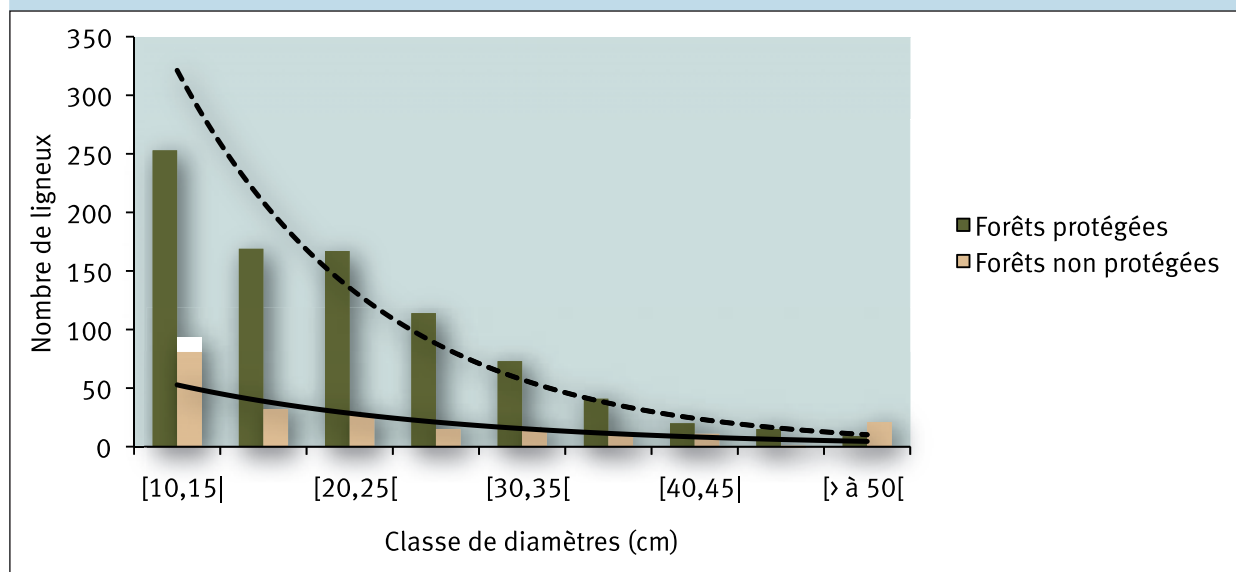

Figure 6.

Distribution des tiges de toutes les espèces ligneuses par classes de diamètre.

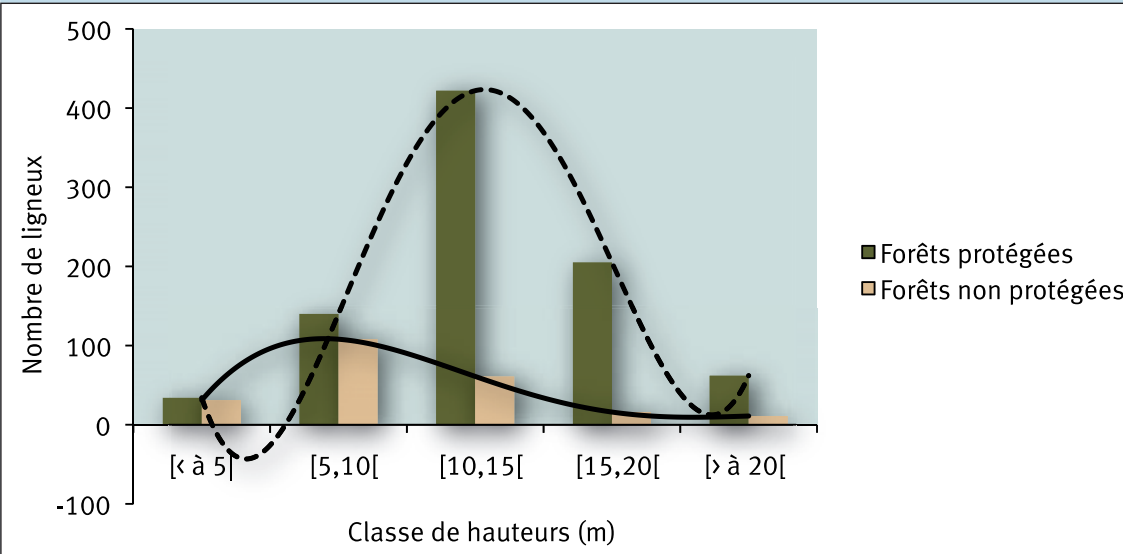

Figure 7.

Distribution des tiges de toutes les espèces ligneuses par classes de hauteur.

tégées ou non (figure 7). La dissymétrie est nettement orientée vers la gauche du graphique (tuteurs pour la culture de l'igname) pour les zones non protégées et montre bien que les grands arbres sont peu nombreux, car ils ont été abattus. Dans les forêts protégées, la dissymétrie est orientée vers la droite, car les grands arbres sont conservés et mieux représentés. Cette distribution des tiges d'arbres par classes de hauteur est mieux ajustée à une fonction polynomiale d'ordre 2 d'équation $y=-71,21 x^{2}$ $+439,39 x-362,2 ; R^{2}=0,75$ pour les forêts protégées et $\mathrm{y}=-11,57 \mathrm{x}^{2}$ $+56,23 x+4 ; R^{2}=0,56$ pour les forêts à accès libre. 


\section{Discussion}

Les forêts claires aux sols fertiles sont convoitées par les agriculteurs (UBOM, ISICHEI, 1995). Outre l'envahissement agricole du domaine soudanien du Togo, elles sont soumises à diverses pressions : les feux, le surpâturage, l'exploitation du bois et le prélèvement des produits forestiers non ligneux, menaces qui pèsent sur leur survie. Mais ces forêts ont des fonctions écologiques, environnementales et fournissent divers produits alimentaires, pharmaceutiques, aromatiques, ligneux, fibreux, ornementaux, fourragers et mêmes toxiques (pour la chasse et la pêche). La forêt constitue aussi un ombrage pour le bétail et un réservoir de protéines (escargots, chenilles, etc.) ainsi qu'un habitat de prédilection pour la grande faune sauvage. Les produits forestiers non ligneux récoltés génèrent des revenus importants qui permettent aux populations locales d'être très peu dépendantes des produits importés trop coûteux (SHARMA et al., 1994). C'est le cas notamment de Parkia biglobosa et de Vitellaria paradoxa qui revêtent une importance socio-économique indéniable (WALA et al., 2005).

De nos jours, le rythme d'exploitation des ressources ligneuses de ces forêts claires risque de compromettre la pérennité des espèces végétales en l'absence de mesures de sauvegarde. Une gestion durable s'avère nécessaire pour les générations présentes et futures. C'est dans cette optique que de nombreux États ont créé des parcs et des réserves de faune pour la protection et l'amélioration de la faune et de la flore. Dans les pays sahéliens, la diversité biologique est conservée dans les réserves naturelles et les parcs nationaux (BELEM, 2001 ; HIEN et al., 2002). C'est le cas du parc national de Fazao-Malfakassa et de la réserve de faune d'Alédjo au Togo, dont les formations ligneuses conservent une richesse spécifique considérable (WoÉGAN, 2007). Des observations analogues sont faites dans les zones protégées de la chaîne de l'Atacora au Bénin par WALA (2004). Au Mali, I. doka, Daniellia oliveri, Anogeissus leocarpus, Pterocarpus erinaceus, Combretum fragans,
Burkea africana et Khaya senegalensis sont importants pour le bien-être des populations qui vivent des ressources offertes par les ligneux (ANDERSON et al., 1994) et d'autres produits forestiers non ligneux, comme les champignons (Yorou et al., 2001). Les forêts claires constituent aussi une source de fourrage pour le bétail en saison sèche. De nombreuses espèces produisent de nouvelles feuilles deux mois avant l'arrivée des pluies. Des études réalisées en Afrique subsaharienne confirment bien que la récolte du fourrage se fait par élagages souvent excessifs, voire étêtages et écimages brutaux par les pasteurs (ANDERSON et al., 1994 ; SINSIN, 2000 ; ONANA, 1998 ; HOUINATO, Sinsin, 2001). Ces pratiques de prélèvement ont aussi un effet négatif sur l'accroissement de ces arbres.

Cette pression anthropique et les évolutions climatiques entraînent une modification de la composition floristique, de la trajectoire naturelle de la dynamique de reconstitution et de régénération des forêts naturelles. Au Togo, l'exploitation anarchique actuelle des forêts claires a un impact négatif sur la conservation de la diversité de ces écosystèmes forestiers. La diversité spécifique, les indices de Shannon et d'équitabilité de Piélou, la densité, la surface terrière, la distribution des tiges par classes de hauteur et de diamètre montrent bien que les forêts protégées sont mieux conservées que celles à accès libre. Ces résultats concordent avec ceux observés dans la chaîne de l'Atacora au Bénin (WALA, 2004). L'impact anthropique modifie négativement la diversité et la structure des forêts perturbées. Malgré ces pressions, les espèces ligneuses des forêts claires à Isoberlinia se régénèrent par semis, par drageons et par rejets de souche, comme le précisent les études de BATIONO (2001), DOURMA (2003), DOURMA et al. (2006), BELLEFONTAINE et al. (2005) et BELLEFontAine (2005). Si les travaux réalisés par DEVINEAU (1999) constatent que le bétail favorise la dissémination des semences de certaines espèces, il est évident que les jeunes semis sont très sévèrement endommagés par le bétail et les feux.
Les espèces ligneuses des forêts claires du domaine soudanien du Togo assurent des fonctions écologiques et environnementales et jouent un rôle alimentaire, pharmaceutique, fourrager, etc. Les produits forestiers non ligneux génèrent des revenus importants. Les enquêtes menées ont montré que les populations riveraines sont conscientes des menaces qui compromettent leur survie. Sur le plan de la structure, les valeurs de la richesse spécifique, de la densité et de la surface terrière dans les forêts claires non protégées sont nettement plus faibles par rapport aux valeurs des forêts protégées. Les structures diamétriques et verticales des forêts protégées sont plus normales.

La situation qui prévaut dans les forêts claires non protégées constitue une menace à court terme pour le maintien de leur durabilité. Le surpâturage et les feux de brousse freinent la croissance des arbres et réduisent souvent à néant la régénération naturelle. Les forêts claires demeurent pour les populations locales une source potentielle de ressources indispensables pour satisfaire leurs besoins quotidiens.

Les plans d'aménagement et de gestion participative, ainsi que le respect intégral des consignes de gestion de ces forêts claires, constituent une priorité absolue pour le gouvernement togolais.

\section{Remerciements}

Les auteurs remercient la Fis (Fondation internationale pour la science) qui a soutenu cette recherche par l'octroi d'une bourse financée par le Comité d'organisation permanent de la Conférence islamique sur la coopération scientifique et technologique (Comstech) et également l'African Forest Research Network (Afornet). 


\section{Références}

ANDERSON J., 1994. Final report. A general overview of the accomplishments of the special technical unit of OADP over the period January 1988 April 1994. Bamako, Mali, ministère du Développement rural et de l'Environnement, service des forêts, unité technique spéciale, $30 \mathrm{p}$.

ANDERSON J., BERTRAND A., KONANDJI H., 1994. Le fourrage arboré à Bamako : production et gestion des arbres fourragers, consommation et filière d'approvisionnement. Sécheresse, 2 (5) : 99-105.

ARBONNIER M., 2009. Arbres, arbustes et lianes des zones sèches d'Afrique de l'Ouest. Mnhn/Quæ, 3e éd., 541 p.

BATIONO B. A., 2001. Régénération naturelle de cinq espèces ligneuses de la forêt classée de Nazinon (Burkina Faso) : Detarium microcarpum Guill. \& Perr., Afzelia africana Sm., Isoberlinia doka Craib \& Stapf, Piliostigma thonningii (Sch.) Miln.Redh. et Terminalia avicennioides Guill. \& Perr. Thèse, Université de Ouagadougou, Burkina Faso, $174 \mathrm{p}$.

BELEM M., 2001. Diversité floristique de deux forêts-galeries de la Réserve de Biosphère de la Mare aux Hippopotames (Burkina Faso, Afrique de l'Ouest). Proceedings of the XVIth AETFAT Conference, National Botanic Garden of Belgium. Systematics and Geography of Plants, 71 : 797-806.

BELLEFONTAINE R., 2005. Pour de nombreux ligneux, la reproduction sexuée n'est pas la seule voie : analyse de 875 cas. Sécheresse (revue électronique), $n^{\circ} 3^{\mathrm{E}}$, décembre 2005.

BELLEFONTAINE R., SABIR M., KOKOU K., GUINKO S., SAADOU M., ICHAOU A., HATEM C., BATIONO B., DOURMA M., KARIM S., 2005. Argumentaire pour l'étude et l'utilisation des marcottes et des drageons dans les pays à faible couvert ligneux. Sécheresse (revue électronique), $\mathrm{n}^{\circ} 3^{\mathrm{E}}$, décembre 2005. http://www.secheresse.info/ article.php3.id_article $=2343$.
DEVINEAU J.-L., 1999. Rôle du bétail dans le cycle culture-jachère en région soudanienne : la dissémination d'espèces végétales colonisatrices d'espaces ouverts (Bondoukuy, sud-ouest du Burkina Faso). Revue d’Écologie (Terre et Vie), 54 (2) : 97-121.

DOURMA M., 2003. Régénération naturelle d'Isoberlinia spp. (Caesalpiniaceae) en zone soudanienne du Togo. Mémoire Dea, Université de Lomé, Togo, $47 \mathrm{p}$.

DOURMA M., GUELLY A. K., KOKOU K., BATAWILA K., WALA K., BELLEFONTAINE R., AKPAGANA K., 2006. Multiplication par drageonnage d'Isoberlinia doka et I. tomentosa au sein des formations arborées au Nord-Togo. Bois et forêts des tropiques, 289 (3) : 49-57.

ERN H., 1979. Die Vegetation Togo. Gliederrung, Gefährdung, Erhaltung. Willdenowia, 9 : 295-312.

GEERLING C., 1982. Guide de terrain des ligneux sahéliens et soudanoguinéens. Wageningen, Pays-Bas, $\mathrm{H}$. Veenman et Zonen B.V., 340 p.

HIEN M., BOUSSIM J., GUINKO S., 2002. L'utilisation de Burkea africana Hook. (Caesalpiniaceae) par les populations d'éléphants Loxodonta africana (Blumen) Bach. dans le Ranch de Gibier de Nazinga (Burkina Faso). Annales de Botanique de l'Afrique de l'Ouest, $2:$ 25-35.

HOUINATO M., SINSIN B., 2001. La pression agro-pastorale sur la zone riveraine de la Réserve de la Biosphère de la Pendjari. Tropicultura, 18 (2) : 112-117.

MAGURRAN A. E., 1988. Ecological diversity and its measurement. Cambridge, Grande-Bretagne, Cambridge University Press, $179 \mathrm{p}$.

ONANA J., 1998. Sur l'utilisation et la régénération naturelle d'Afzelia africana Smith ex Person. Le Flamboyant, $46: 33$.

OURO-DJÉRI E., 1994. Approvisionnement et consommation des combustibles ligneux à Sokodé (Togo). Mémoire, Université de Dschang, Cameroun, 96 p.
SHARMA N. P., RIETBERGEN S., HEIMO C. R., PTEL J., 1994. A strategy for the forest sector in sub-saharan Africa. Washington, USA, World Bank, Africa Technical Department series, Technical Paper 251, 69 p.

SINSIN B., 1997. La transhumance dans les aires protégées d'Afrique de l'Ouest. Revue d'information, Pacipe (Cotonou, Bénin), 5 : 4-14.

SINSIN B., 2000. Stratégie d'adaptation du système d'élevage bovin aux pénuries fourragères chez les Bétamaribé du Bénin. Berichte des Sonderforschungsbereichs, 268, 14 : 209-222.

UBOM R. M., ISICHEI A. O., 1995. Soil vegetation interrelationships in Isoberlinia woodlands in Northwestern Nigeria. Acta Botanica Hungarica, 33 (3-4) : 289-301.

WALA K., 2004. La végétation de la chaîne de l'Atakora au Bénin : diversité floristique, phytosociologie et impact humain. Thèse de doctorat, Université de Lomé, Togo, 138 p.

WATSON L., DALLWITZ M. J., 1993. The genera of Leguminosae-Caesalpinioideae and Swartzieae : descriptions, identification and information retrieval. French translation by E. Chenin. Version : 22 mars 2009. http://delta-intkey.com.

WALA K., SINSIN B., GUELLY K. A., KOKOU K., AKPAGANA K., 2005. Typologie et structure des parcs agroforestiers dans la préfecture de Doufelgou (Togo). Sécheresse, 16 (3) : 209-216.

WHITE F., 1986. La végétation d'Afrique. Mémoire, carte de la végétation d'Afrique. Unesco/Aetfa/Unso, 383 p.

WOÉGAN Y. A., 2007. Diversité des formations végétales ligneuses du parc national de Fazao-Malfakassa et de la réserve de faune d'Alédjo (Togo). Thèse de doctorat, Université de Lomé, Togo, $142 \mathrm{p}$.

YOROU S. N., DE KESEL A., SINSIN B., CODJIA J. T. C., 2001. Diversité et productivité des champignons comestibles de la forêt classée de WariMaro (Bénin, Afrique de l'Ouest). Systematics and Geography of Plants, 71 (2) : 613-625. 